\title{
Finite element analysis of plasticity-induced fatigue crack closure using contact elements
}

\author{
M. G. Gökçen ${ }^{1} \&$ V. Kalenderoğlu ${ }^{2}$ \\ ${ }^{1}$ Department of Mechanical Engineering, Doğuş University, Turkey \\ ${ }^{2}$ Department of Mechanical Engineering, Boğaziçi University, Turkey
}

\begin{abstract}
To predict crack opening stresses, contact elements are incorporated into the elastic-plastic finite element analysis of fatigue crack closure. A code using ANSYS Parametric Design Language (APDL) is developed. In spite of the fact that the use of contact elements in modelling crack surface contact and crack closure is inherently natural, efforts to incorporate them in the finite element analysis of crack closure are not widespread. The traditional method of modelling crack closure is based on placing truss elements at the crack surface nodes. In the present research, contact elements are used to model crack surface contact. While the load is applied incrementally, crack opening stress is determined by monitoring the state of the contact elements. The results of two-dimensional plane strain finite element analyses are in good agreement with previous work reported in the literature. Instead of finding crack opening stress at every load cycle, an algorithm to find crack opening stress at predetermined load cycle intervals is developed. With the developed algorithm it was possible to analyze crack closure behaviour during a larger number of load cycles with less execution time. The algorithm that is implemented is parametrically analysed. The effect of load increment is investigated. Since crack opening stress is not evaluated at every cycle, the effect of how often opening stresses are determined is another issue that is investigated. As a result of the simulation with a relatively high number of cycles, it was possible to observe the final stabilization in the crack opening stress values that follows a decay after the initial plateau.
\end{abstract}

Keywords: fatigue crack closure, plasticity induced closure, finite element analysis, contact elements. 


\section{Introduction}

Elber [1] demonstrated the importance of plasticity induced crack closure, which is shown to be the result of residual deformations in the wake of a growing crack. Although there exists other closure phenomena, such as roughness induced crack closure and oxide induced crack closure, they are often assumed to be secondary.

In plasticity induced crack closure, the crack growth is governed by the effective stress intensity range, $\Delta \mathrm{K}_{\text {eff. }}$. While fatigue crack propagates, two different types of crack tip plastic zones are generated, fig. 1. The forward plastic zone is associated with the plastic deformation of the material near the crack tip at maximum load. The second zone of interest is the reversed plastic zone, which is associated with the compressive yielding of the material near the crack tip at minimum load.

Many researchers have carried out finite element analyses to simulate plasticity-induced fatigue crack closure. Traditional studies employ the following basic algorithm. A mesh is created with a suitably refined region near the crack front. Since plastic deformations occur in the vicinity of the crack tip, an elasticplastic material model is employed. Remote tractions are then applied to the model and cycled between a maximum stress, $\mathrm{S}_{\max }$, and a minimum stress, $\mathrm{S}_{\min }$. At each cycle, nodes at the crack front are released and thus the crack is allowed to advance one elemental length, da. The crack that propagates through the plastic zone leads to the formation of a plastic wake. Crack surface nodes are monitored to detect contact between crack faces, and thus predict crack closure.

To prevent the crack surface penetration at minimum load a mechanism must be implemented. This can be achieved by using contact elements. Since contact elements may lead to convergence problems and generally involve long execution times, their incorporation into the crack closure analysis was not preferred in the past [2]. Newman [3] implemented spring elements to simulate crack surface contact behaviour. A spring element was connected to each boundary node on the crack surface. For open nodes, the spring stiffness was set equal to zero, and for closed nodes the stiffness was assigned a large value. Skinner and Daniewicz [2] proposed another approach. During loading and unloading, they monitored stresses and displacements along the crack surface. A negative nodal displacement indicated that the crack was closed at this point, and

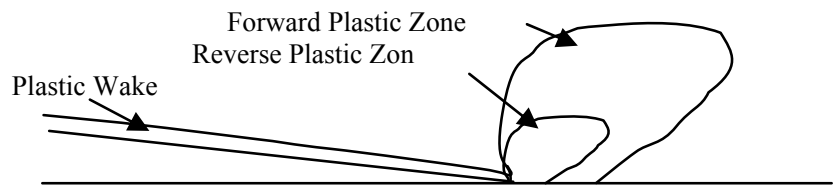

Figure 1: $\quad$ Plastic deformation around a growing crack. 
(a) Normalized opening stress, $\mathrm{S}_{\mathrm{op}} / \mathrm{S}_{\mathrm{y}}$

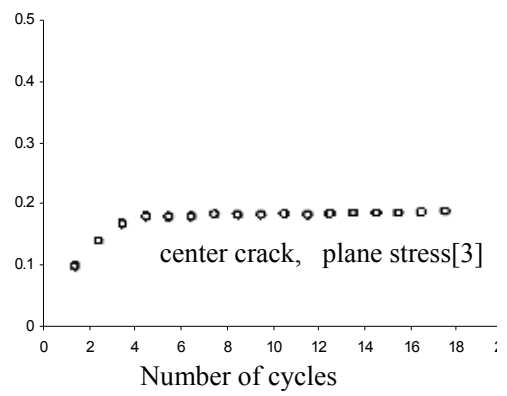

(b) Normalized opening stress, $S_{o p} / S_{\max }$

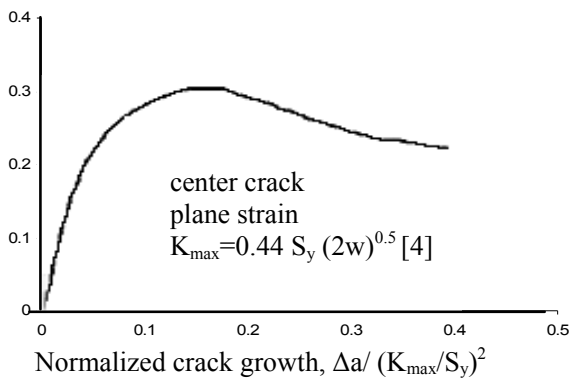

Figure 2: $\quad$ Stabilization of crack opening stress values under plane-stress and plane-strain, where $\mathrm{S}_{\mathrm{y}}$ is material yield stress, and w is half-width.

the displacement of that node was set equal to zero. In subsequent load increment, when a tensile nodal stress is encountered at a constrained node, the node is released. The load level at which all the constrained nodes of the crack surface are released is taken as the crack opening stress value.

Under constant amplitude loading, the crack opening stress will typically increase monotonically with increasing crack length until a stabilized value is reached, fig. 2 .

McClung et al. [4] have shown that under constant amplitude loading conditions, the crack should propagate completely through the initial forward plastic zone in order to form a stabilized plastic wake. The results of Newman [3] for plane stress analyses are shown in fig. 2a. The results of Fleck and Newman [5] for plane strain analyses are shown in fig. 2b. Lee and Song [6], on the other hand, reported that for a stabilized crack opening level under plane strain conditions, the crack must be advanced through approximately four times the initial monotonic plastic zone. At what load crack should be advanced is another issue. To accelerate convergence some researchers suggest that crack advance should take place at minimum load $[7,8]$. Since in reality, there are no mechanism present to cause crack growth when the crack is closed other researchers suggest that crack advance should take place at maximum load [2, 3, $9,10]$. This approach is also adopted in this work.

\section{Finite element model}

In the present research, the model of Skinner and Daniewicz [2] is modified to implement contact elements in the prediction of plasticity induced fatigue crack closure. Rather than evaluating the crack opening stresses at each cycle an algorithm is developed to determine those stresses at predefined intervals.

The approach is actually simple: the structure is loaded in cycles alternating between maximum stress and minimum stress. After maximum stress is applied the crack is advanced by one element size, da. 

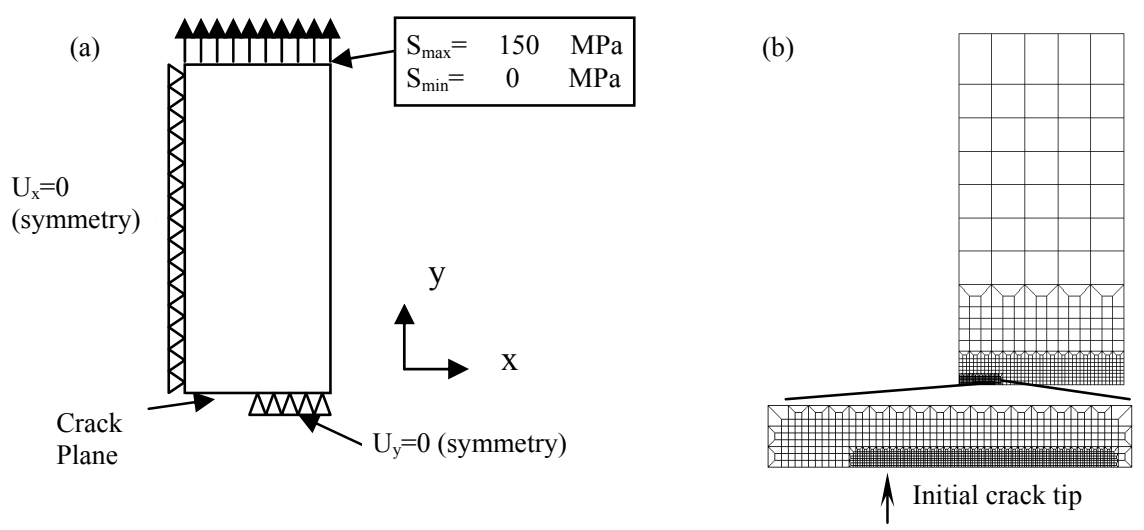

Figure 3: (a) Schematics of the boundary conditions and loading; and (b) finite element mesh.

A two dimensional plane strain model is created with half-width $\mathrm{w}=230 \mathrm{~mm}$, half height $\mathrm{h}=460 \mathrm{~mm}$ and crack length $\mathrm{a}=0.0279 \mathrm{~mm}$. This specimen is first modelled by Newman [3]. The boundary conditions and the loading are schematically shown in fig. 3a. Elastic perfectly plastic material is assumed. The modulus of elasticity $\mathrm{E}$ is $70 \mathrm{GPa}$, and yielding stress Sy is $350 \mathrm{MPa}$. Linear four noded solid elements are used. The mesh used in this work is shown in Figure 3 b. To model the contact, point to surface contact elements are used.

The mesh size criteria used is $\Delta \mathrm{a} / \mathrm{r}_{\mathrm{f}} \leq 0.1$ is used. Forward plastic zone size, $\mathrm{r}_{\mathrm{f}}$, is calculated by the method proposed by McClung and Sehitoglu $[11,12]$ as

$$
\frac{r_{f}}{a}=\left(\frac{S_{\max }}{S_{y}}\right)^{2}
$$

where $r_{f}$ is the forward plastic zone size, $S_{\max }$ is the maximum stress, and $S_{y}$ is the material yield stress. In the present model da is selected as $0.18 \mathrm{~mm}$.

\subsection{Crack closure model}

The crack is modelled by constraining the nodes in y-direction on the crack surface and in the uncracked region. The evolution of the plastic wake can be simulated by advancing the crack tip through the monotonic plastic zone.

At maximum load, crack is advanced gradually rather than allowing it grow instantaneously by direct removal of the displacement constraints of the crack tip node. To achieve such a gradual growth, following the removal of the displacement constraint of the crack tip node, a force equal to a fraction of the reaction force calculated at the node in the presence of the displacement constraint is applied. This is done in the first load step of the incremental loading scheme. The applied force is than reduced to zero in the subsequent three load steps. At the end of the fourth load step the node becomes free and the crack advances one elemental length. 


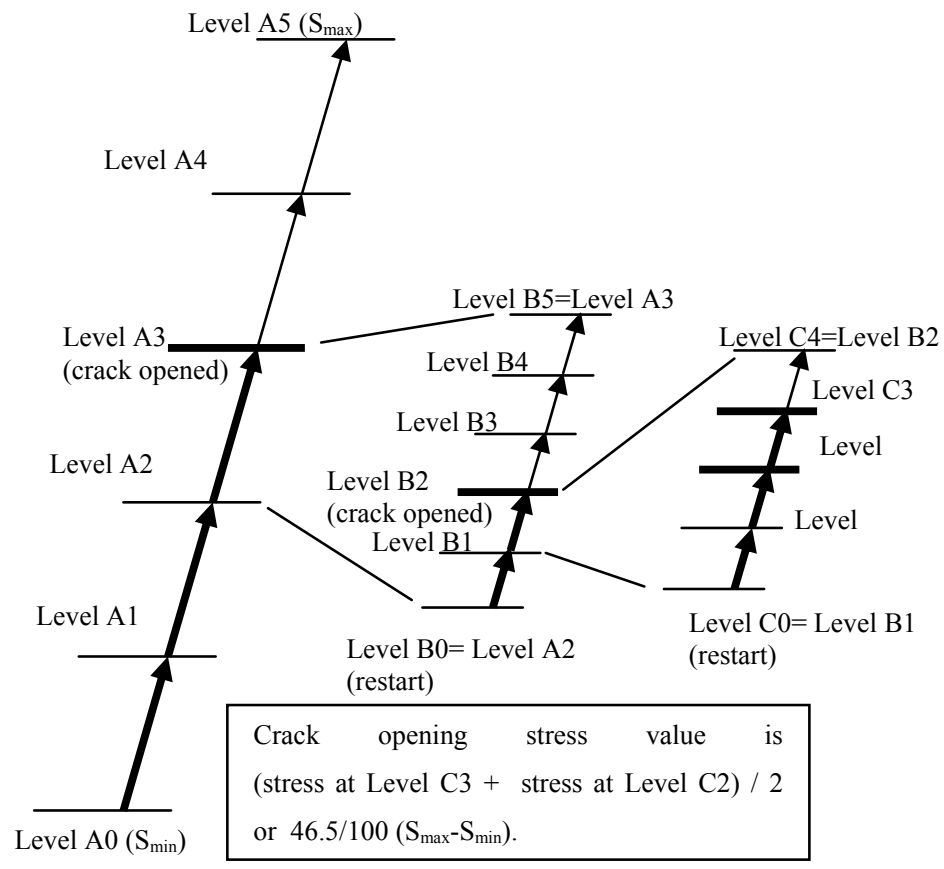

Figure 4: Schematic presentation of the determination of opening stress during loading.

The algorithm implemented to find the crack opening stress is schematically illustrated in fig. 4. First, the maximum load is divided into five increments and the load is applied incrementally. At the end of each load level the status of the contact elements is monitored. For a contact element the normal force is equal to zero if the node and the surface defining the element are not in contact. In case of contact between node and surface, a non-zero normal force prevents penetration of the node into the surface. If at the end of a load increment contact element normal forces do not add up to zero next load increment is applied. If the resultant normal force of all the contact elements is zero, the crack is considered to be open. This load level corresponds to level A3, fig. 4. It then follows that crack opening has occurred somewhere between level A2 and A3. Now the load increment between level $\mathrm{A} 2$ and $\mathrm{A} 3$ is further subdivided into five increments. The specimen is loaded starting at level A2. This level is identified as Level B0, fig. 4. Proceeding similarly, load is applied incrementally till crack opening is detected. This occurs at level B2, fig. 4, with the implication that crack is opened somewhere in between level B1 and B2. Now the load increment between level B1 and B2 is further subdivided into four increments, and the specimen is loaded restarting at level $\mathrm{B} 1$, which is identified as Level $\mathrm{C} 0$, fig. 4. Load is applied incrementally till crack opening is detected. This occurs at level C3, fig. 4, with the implication that crack is opened somewhere in between level C2 and C3. Crack opening stress is assumed to occur at the halfway between the last level 
and the level that precedes it. These levels correspond to level $\mathrm{C} 2$ and C3, fig. 4. The crack opening stress turns out to be the mean of the stresses at level $\mathrm{C} 2$ and C3 or $46.5 / 100\left(\mathrm{~S}_{\max }-\mathrm{S}_{\min }\right)$, fig. 4. With this incremental loading scheme it is possible to determine the crack opening stress with a resolution of $1 / 100\left(S_{\max }{ }^{-}\right.$ $\mathrm{S}_{\min }$ ). The same resolution could have been obtained by dividing the load span $\left(\mathrm{S}_{\max }-\mathrm{S}_{\min }\right)$ into 100 increments and loading incrementally with this load increment. With the algorithm implemented in the present work the same result is attained to with only eight load steps. This is one of the prominent features of the developed algorithm.

Another feature of the developed algorithm is its ability to predict the crack opening stresses at predetermined number of load cycles. This feature allows one to increase the total number of simulated cycles with less execution time. The idea is simply to determine the crack opening stress at predetermined number of load cycles rather than evaluating it at every cycle, fig. 5. The maximum and minimum loads are applied in cycles, and at every maximum load the crack is advanced by one elemental length, da, without determining the crack opening stress value. The load step in each cycle is taken as the one fifth of the load span $\left(\mathrm{S}_{\max }-\mathrm{S}_{\min }\right)$. At the beginning of the predefined load cycle at which the crack opening stress is to be determined, the current state of the analysis is saved for later restart. At this stage, the algorithm for the determination of crack opening stress value is executed and the crack opening stress is determined. With the determination of the crack opening stress the algorithm stops, records the crack opening stress value and the saved state at the beginning of the load cycle is recovered. Here on, load cycling continues without determining the crack opening stress values until the next predetermined load cycle, for which the crack opening stress to be determined, is reached. This algorithm reduces the execution time appreciably for the same number of cycles if the predetermined load cycle interval is large. If the predetermined number of load cycles at which the crack opening stress is evaluated is one, the execution time in the present algorithm, although less, is comparable with that of traditional methods reported in the literature, typically less than by five per cent.

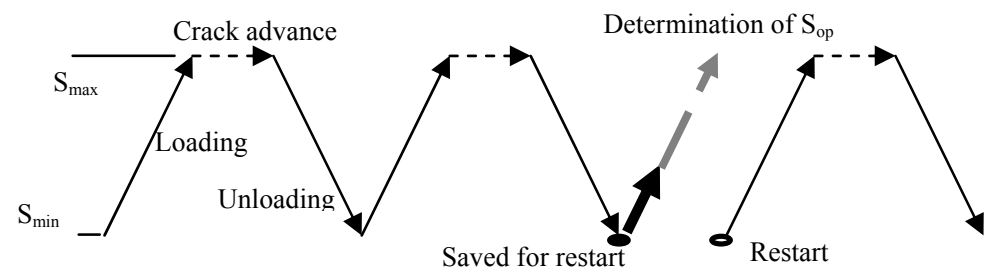

Figure 5: Schematic presentation of how the algorithm for the determination crack opening stress, $\mathrm{S}_{\mathrm{op}}$, value is incorporated in to the load cycles. 


\section{Results}

\subsection{Plane strain analysis results}

\subsubsection{Crack opening stress}

The results of the finite element analysis of fatigue crack closure using contact elements are given in fig. 6 . The crack opening stress is determined at every cycle for twenty cycles and then at every fifth load cycle until hundred cycles is completed. The plateau at which crack opening stress stabilizes as the crack passes through the initial forward plastic zone can easily be observed. The crack opening stress decreases after the initial plateau and stabilizes at a level lower than that of plateau. This stabilized level is reached when the crack is advanced approximately four times the initial plateau is reached. This result is in agreement with the observations of Lee and Song [6].

Since the crack opening stress values are calculated at cyclic intervals rather than evaluating at each cycle, it was possible to observe the crack opening behaviour for much higher number of load cycles with appreciably less execution time. The execution time for ten load cycles required by the present model with crack opening stress values evaluated at every fifth cycle is

(a)

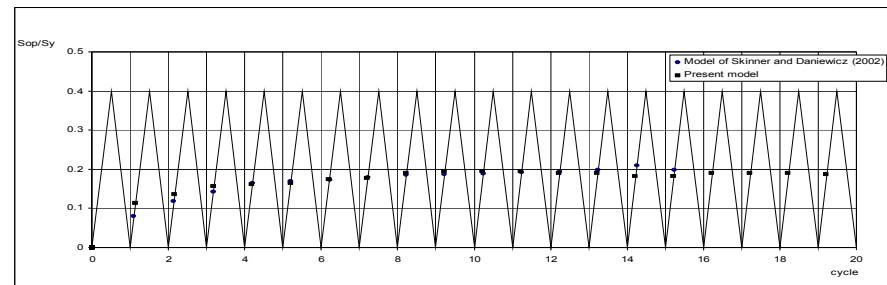

(b)

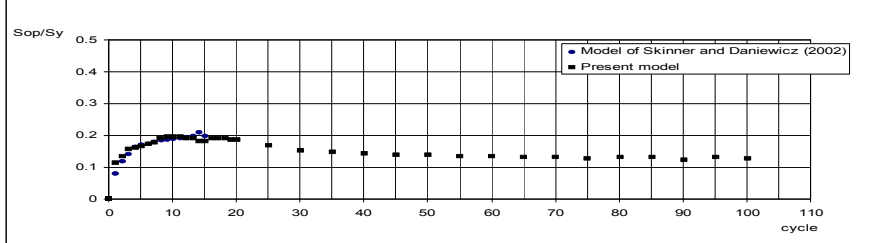

Figure 6: Normalized crack opening stress values (a) for the first twenty load cycles and (b) for one hundred load cycles.

Table 1: $\quad$ Execution time comparison.

\begin{tabular}{|c|c|c|c|}
\hline \multicolumn{4}{|c|}{ Time required for 10 load cycles } \\
\hline & $\begin{array}{c}\text { Model of } \\
\text { Skinner and } \\
\text { Daniewicz [2] }\end{array}$ & $\begin{array}{c}\text { Present model } \\
\text { with } 1 / 100\left(S_{\max }-S_{\min }\right) \text { load } \\
\text { increments in finding } S_{\text {op }}\end{array}$ & $\begin{array}{c}\text { Present model } \\
\text { with } 1 / 200\left(S_{\max }-S_{\min }\right) \\
\text { load increments }\end{array}$ \\
\hline every cycle & $36 \min 26 \mathrm{sec}$ & $29 \mathrm{~min} 17 \mathrm{sec}$ & $34 \mathrm{~min} 45 \mathrm{sec}$ \\
\hline every fifth cycle & - & $12 \mathrm{~min} 28 \mathrm{sec}$ & $16 \mathrm{~min} 22 \mathrm{sec}$ \\
\hline every tenth cycle & - & $9 \mathrm{~min} 57 \mathrm{sec}$ & $13 \mathrm{~min} 4 \mathrm{sec}$ \\
\hline
\end{tabular}


approximately one third of the execution time required by the method of Skinner and Daniewicz [2]. The execution times for a ten cycle simulation with different schemes of determining crack opening stress values are listed in table 1.

\subsubsection{Crack profile and contact stress}

The change in the crack surface profile is monitored. Crack surface profiles at minimum and maximum loads for the first, twentieth and hundredth load cycles are plotted in fig. 7. The effect of the hump is clearly seen in the hundredth load cycle. The crack closes behind the crack tip and manifests the typical plasticity induced crack closure behaviour. The normal stress values of the contact elements at minimum loads are shown in fig. 8 for the first and hundredth load cycles, respectively. The normal stress values can also be referred to as the contact stress. Note that in these graphs, the upper half of the plot shows the crack surface profile while the lower half of the plot shows the contact stress values.

\subsection{Plane stress analysis results}

Analogues to the results of the plane strain analyses. Crack opening stresses are shown in fig. 9, while crack profile and contact stresses are shown in fig. 10. The hump that was under plane strain stress conditions did not develop in the case of plane stress.
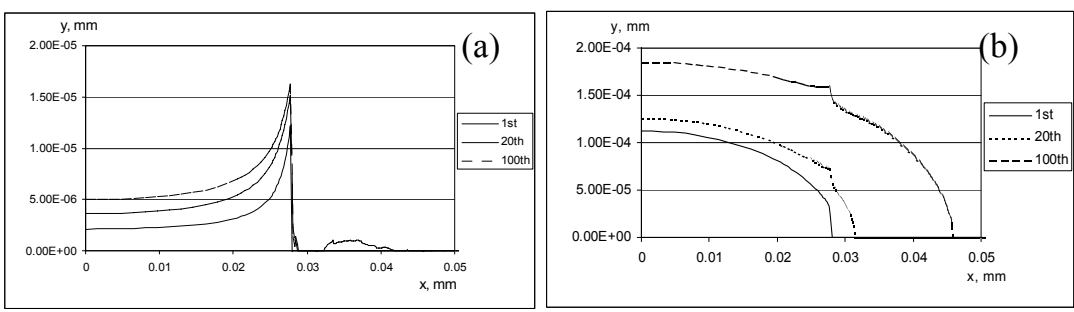

Figure 7: Crack surface profiles for the first, twentieth and hundredth load cycles (a) at maximum load and (b) at minimum load.

(a)

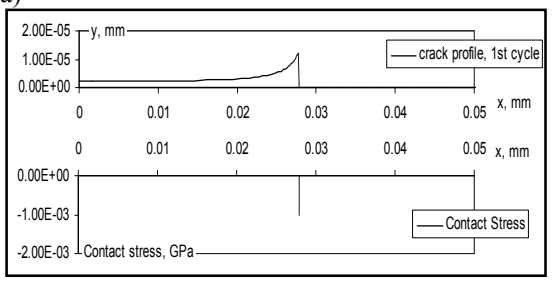

(b)

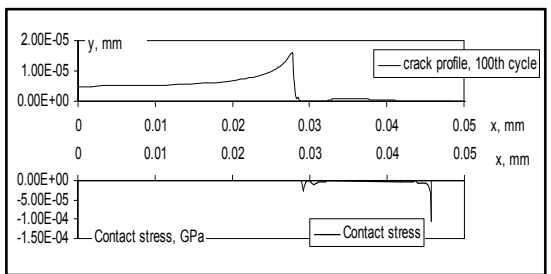

Figure 8: The crack surface profile and the contact stress values for minimum load (a) at first load cycle and (b) at hundredth load cycle. 


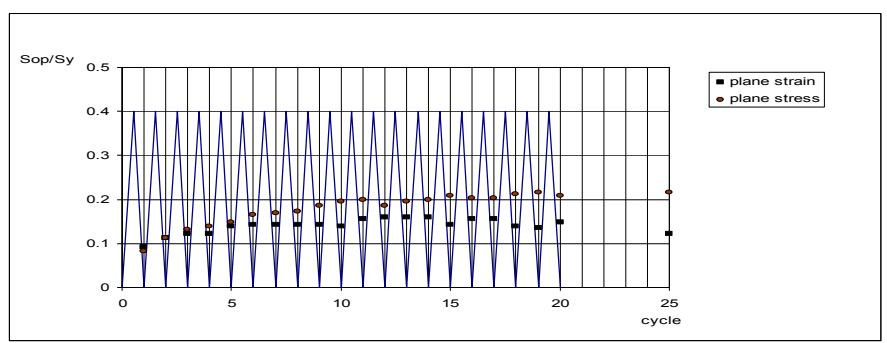

Figure 9: Comparison of the normalized crack opening stress values for plane strain and plane stress configurations for the first twenty five load cycles.

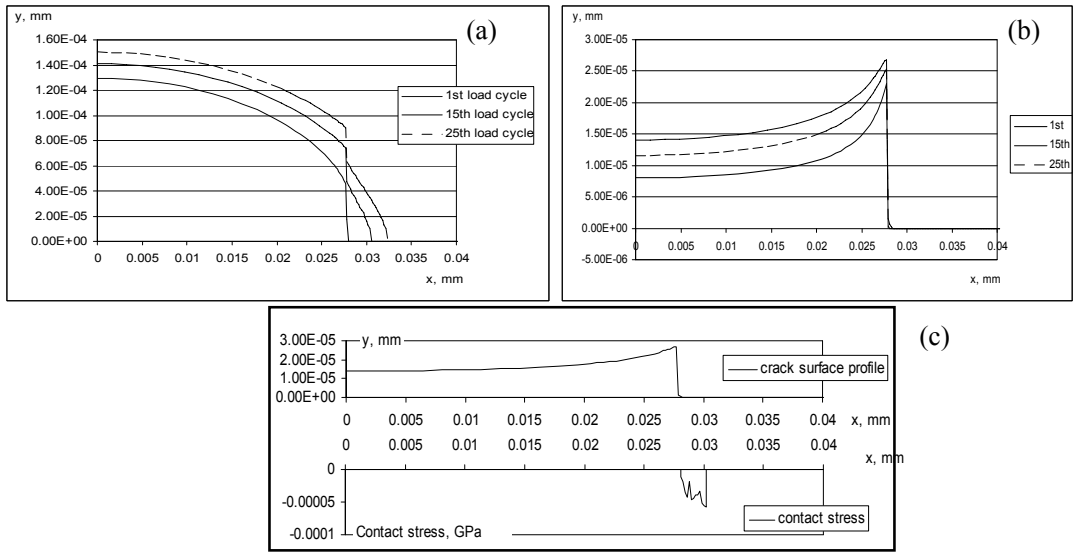

Figure 10: Crack surface profiles for the first, and twenty-fifth load cycles for plane stress (a) at maximum load and (b) at minimum load. (c) The crack surface profile and the contact stress values for minimum load at the twenty-fifth load cycle.

\subsection{Parametric analysis of the algorithm}

Sensitivity of crack opening stress prediction to the parameters referred to as the predetermined number of load cycles at which crack opening is evaluated, to crack opening stress resolution and to the scheme of advancing the crack is investigated. The results presented here are for the plane strain analysis. The load cycle interval size on the crack opening stress does not have an effect on the values of the crack opening stress. The effect of the resolution in determining the crack opening stress is investigated by varying the number of divisions into which the load span $\left(\mathrm{S}_{\max }-\mathrm{S}_{\min }\right)$ is divided. Figure 11 shows the results for 10 5000 divisions. It can then be concluded that as long as the number of divisions is not as low as ten crack opening stress prediction is reasonably insensitive to the number of load span divisions. The crack is advanced at maximum load by releasing the crack tip node gradually. The gradual release was achieved by 


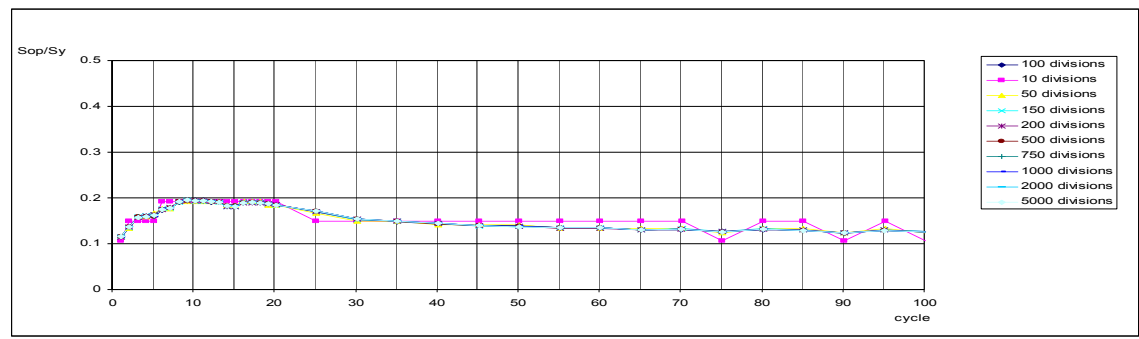

Figure 11: The effect of resolution of the crack opening stress value evaluation for one hundred cycles.
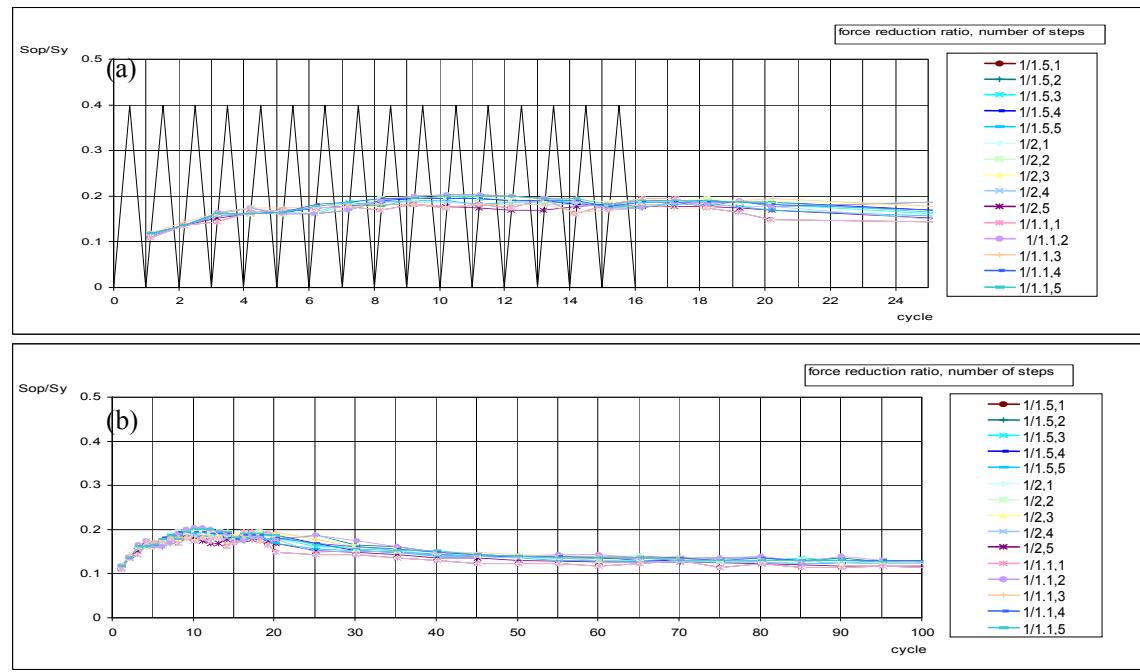

Figure 12: Effect of crack advance parameters: force reduction ratio (varies between 1/1.1 and 1/2) and number steps (varies between 1 and 5). (a) for the first twenty load cycles, (b) for one hundred load cycles.

applying a fraction of the reaction force that was calculated in the presence of the displacement constraint. The applied force is than incrementally reduced to zero. The parameters involved in this scheme are the force reduction ratio and the number of step in reducing the applied load to zero. The force reduction ratio is varied between $1 / 1.1$ and $1 / 2$ and the number of steps is varied between 1 and 5 , fig. 12. It can be seen that the results are rather insensitive to force reduction ratio and to the number of steps in which the force is reduced to zero.

\section{Conclusion}

Contact elements are implemented into the finite element analysis of fatigue crack closure to predict crack opening stress. Using ANSYS Parametric Design 
Language (APDL), a finite element code is developed. A parametric analysis of the newly developed algorithm is carried out.

To determine crack opening stress at predetermined load cycles, a new algorithm is developed. The new analysis provides the capability of evaluating crack opening stress at cyclic intervals rather than evaluating crack opening stress at each cycle. It is therefore possible to observe the crack opening behaviour for a prolonged period with appreciably less execution time. While the execution times of both the method of Skinner and Daniewicz [2] and the present method in evaluating crack opening stress at every load cycle are comparable, the execution time for ten load cycles required by the present method with crack opening stress values evaluated at every fifth cycle is approximately one third of the execution time required by the method of Skinner and Daniewicz [2]. This algorithm reduces the execution time appreciably for the same number of cycles if the predetermined number of load cycles is large.

With the capability of observing crack closure behaviour for a large number of load cycles, typically hundred, it was observed that following an initial stabilization after the crack propagated through initial forward plastic zone, a subsequent decay and further stabilization occurred. The final stabilized level is reached when the crack is advanced approximately four times the length in which the initial plateau is reached. This result is in agreement with the observations of Lee and Song [6].

Plane stress analyses are carried out and are compared with the plane strain analysis results. As expected, the plane stress crack opening stresses are higher and stabilize earlier.

Plasticity induced fatigue crack closure occurs because of the plastic deformation near the crack tip. The crack surface profiles, both at maximum load and minimum load, are plotted as the crack is advanced. In plane strain, the hump generated by the initial load cycle is the main cause of crack closure. In plane stress the closure occurs much close to the crack tip as compared to the plane strain situation. Normal stresses calculated at the contact elements support this observation.

The newly introduced parameters related to the implementation of contact elements are investigated. The effect of the load application increments, which can be thought of as resolution, has negligible effect on the crack opening stress determination. The load cycle interval size is seen to have no effect on the results, as well.

As a further research the analysis can be extended to model three-dimensional fatigue crack closure. With the capability of simulating high number of load cycles, the present model can also be used as a tool for the investigation of the effects of overloads in fatigue crack growth.

\section{References}

[1] Elber, W., Fatigue Crack Closure under Cyclic Tension. Engineering Fracture Mechanics, 2, pp. 37-45, 1970. 
[2] Skinner, J.D. \& Daniewicz, S.R., Simulation of Plasticity-Induced Fatigue Crack Closure in Part-Through Cracked Geometries Using Finite Element Analysis. Engineering Fracture Mechanics, 69, pp. 1-11, 2002.

[3] Newman, J.J.C., Finite-Element Analysis of Crack Growth under Monotonic and Cyclic Loading. Cyclic Stress-Strain and Plastic Deformation Aspects of Fatigue Crack Growth, ed. L.F. Impellizzeri, ASTM STP 637: Philadelphia, pp. 56-80, 1977.

[4] McClung, R.C., Thacker, B.H. \& Roy, S., Finite Element Visualization of Fatigue Crack Closure in Plane-stress and Plane-strain. International Journal of Fracture, 50, pp. 27-49, 1991.

[5] Fleck, N.A. \& Newman, J.C., Analysis of Crack Closure under Plane-strain Conditions. Mechanics of fatigue crack closure, eds Newman, J.J.C. \& Elber, W., ASTM STP 982: Philadelphia, pp. 319-341, 1988.

[6] Lee, H.J. \& Song, J.H., Finite Element Analysis of Fatigue Crack Closure under Plane Strain Conditions: Stabilization Behaviour and Mesh Size Effects. Fatigue and Facture in Engineering Materials and Structures, 28, pp. 333-342, 2005.

[7] Wu, J. \& Ellyin, F.A., Study of Fatigue Crack Closure by Elastic-Plastic Finite Element Analysis for Constant-Amplitude Loading. International Journal of Fracture, 82, pp. 43-65, 1996.

[8] Heper, R. \& Vardar, O., Elastic-plastic Material Response of Fatigue Crack Surface Profiles due to Overload Interactions. International Journal of Fracture, 25(9-11), pp. 801-810, 2003.

[9] Blom, A.F. \& Holm, D.K., An Experimental and Numerical Study of Crack Closure. Engineering Fracture Mechanics, 22, pp. 997-1101, 1985.

[10] Pommier, S., Plane Strain Crack Closure and Cyclic Hardening. Engineering Fracture Mechanics, 69, pp. 25-44, 2001.

[11] McClung, R.C. \& Sehitoglu, H., On the Finite Element Analysis of Fatigue Crack Closure-1: Basic Modeling Issues. Engineering Fracture Mechanics, 33(2), pp. 237-252, 1983.

[12] McClung, R.C. \& Sehitoglu, H., On the Finite Element Analysis of Fatigue Crack Closure-1: Basic Modeling Issues. Engineering Fracture Mechanics, 33(2), pp. 253-272, 1983. 